\title{
MINIMIZAÇÃO DO CUSTO DO SERVIÇO DE POTÊNCIA REATIVA FORNECIDA PELOS GERADORES LEVANDO EM CONTA A RESERVA DE POTÊNCIA: UMA ABORDAGEM EVOLUTIVA
}

\author{
Yuri Molina Rodríguez* \\ percy@dee.ufma.br
}

\author{
Osvaldo R. Saavedra* \\ osvaldo@dee.ufma.br
}

\author{
Ricardo B. Prada ${ }^{\dagger}$ \\ pradadele.puc-rio.br \\ *Departamento de Engenharia de Eletricidade \\ Universidade Federal de Maranhão \\ São Luís, Maranhão, Brasil \\ ${ }^{\dagger}$ Departamento de Engenharia Elétrica \\ Pontifícia Universidade Católica \\ Rio de Janeiro, Brasil
}

\begin{abstract}
Voltage/reactive power problems can occur at any time during system operation, due to contingencies and/or to demand changes. Therefore, the ancillary service for reactive power is necessary to improve the operating state avoiding system security and reliability degradation. Control is to act in relatively small time periods. Such a service is to be acquired by the System Independent Operator from agents connected to the network, such as synchronous generators and compensators, and static VAR compensators.
\end{abstract}

This paper proposes a methodology for minimizing the cost of the reactive power service provided by generators considering the coupling among it, the power reserve service and the energy market. The "allocated reserve" payment method for the reserve power service was used. Numerical results with the IEEE 14-Bus and IEEE 30-Bus test-systems

ARTIGO CONVIDADO:

Versão completa e revisada de artigo apresentado no SBAI-2005

Artigo submetido em 05/06/2006

1a. Revisão em 22/08/2006

2a. Revisão em 09/11/2006

Aceito sob recomendação do Editor Convidado

Prof. Osvaldo Ronald Saavedra Mendez are presented in order to illustrate the mentioned coupling as well as its economic implications.

KEYWORDS: ancillary service, reactive power support, power reserve, power market, evolutionary computation.

\section{RESUMO}

$\mathrm{Na}$ operação dos sistemas de transmissão de potência, os problemas de tensão/potência reativa podem ocorrer a qualquer momento devido a contingências e/ou alterações na demanda. Portanto, são necessários serviços ancilares de suporte de potência reativa em períodos de tempo relativamente pequenos para não comprometer a segurança e a confiabilidade do sistema elétrico. Esse serviço pode ser contratado pelo Operador Independente do Sistema a agentes conectados ao sistema elétrico, tais como geradores e compensadores síncronos, e compensadores estáticos.

Este artigo propõe uma metodologia para minimizar o custo do serviço de potência reativa provida por geradores considerando o acoplamento com o serviço de reservas de potência e com o mercado de energia. Considera-se o método 
de pagamento de "reserva atribuída" para o serviço de reserva de potência. Reportam-se resultados com os sistemas-teste IEEE 14-barras e IEEE 30-barras, ilustrando o acoplamento mencionado, assim como as implicações econômicas.

PALAVRAS-CHAVE: serviços ancilares, suporte de potência reativa, reserva de potência, mercado de energia, computação evolutiva.

\section{INTRODUÇÃO}

Nestes últimos anos, os sistemas elétricos de potência têm experimentado mudanças estruturais importantes, passando de um ambiente regulado de estrutura vertical para um ambiente desregulado e descentralizado. Em qualquer um dos ambientes citados, o principal objetivo é a garantia de um sistema de potência operando em forma eficiente e dentro de níveis adequados de confiabilidade. Para atender este objetivo é necessária a ação de serviços complementares. Em particular, o serviço ancilar de suporte de potência reativa tem um papel importante no sistema de transmissão, através da manutenção apropriada do perfil de tensão. Este serviço ancilar torna-se especialmente importante para enfrentar possíveis contingências.

Em um sistema de potência, manter o módulo da tensão dentro de um limite especificado em todas as barras é um requisito básico e indispensável (já que os equipamentos a elas conectados foram projetados para trabalhar com tensão nominal). Assim, a monitoração e controle do perfil de tensão beneficiam o sistema, por exemplo, minimizando as perdas ativas, aumentando a capacidade de transmissão, lidando com congestionamentos na transmissão. Devido à forte ligação entre a potência reativa e o módulo da tensão, o meio mais utilizado para manter o perfil de tensão é o suporte de potência reativa. A compensação de potência reativa é feita através do uso de diversos equipamentos, tais como capacitores e/ou reatores em paralelo, compensadores síncronos e estáticos .

Os dispositivos que fornecem suporte de potência reativa têm características diferentes em termos de velocidade de resposta, custo do capital, custos de operação e custos de oportunidade. Por exemplo, os geradores podem mudar seu nível de fornecimento e absorção de potência reativa com maior rapidez que outros equipamentos de controle de tensão. Porém, terá um alto custo de oportunidade se o fornecimento de potência ativa tiver que ser reduzido para gerar mais potência reativa. O custo de oportunidade é definido economicamente como o benefício que se perde por não usar-se um recurso da melhor maneira possível (Zhong, 2003), (Bhattacharya and Zhong, 2001).

Os geradores, além de produzir potência ativa para o mercado de energia, fornecem também a reserva de geração para a regulação primária e secundária para o mercado de reserva (existente em vários sistemas). Desta forma, um determinado gerador pode participar em três mercados diferentes. Porém, as ofertas são interdependentes devido à sua capacidade física.

Da perspectiva dos proprietários dos geradores, uma compensação financeira adequada para o suporte de potência reativa e reserva de potência, forneceria um sinal claro de mercado para eles equilibrarem as necessidades de geração de potência ativa e reativa (Kundur and Zhang, 2001).

Do ponto de vista de um gerador, o custo de oportunidade pode ser interpretado como o acoplamento entre o mercado de energia e o serviço ancilar de potência reativa. Por causa do custo de oportunidade, o custo do suporte de potência reativa é altamente não-linear com respeito à demanda anual. O custo deste serviço aumenta lentamente com o incremento da demanda de energia (pouco acoplamento). Porém, à medida que se aproxima do pico de demanda anual, o custo de oportunidade domina rapidamente o custo total (alto acoplamento) (Gross and Tao, 2002), (Hirst and Kirby, 1997).

Neste trabalho, propõe-se um método para minimizar o custo do serviço de suporte de potência reativa quando fornecido por geradores, considerando o acoplamento com o serviço de reserva de potência e com o mercado de energia. O procedimento permite auxiliar o agente gerador no processo de tomada de decisão para ofertar seus produtos no mercado, visando uma solução mais rentável. O método de pagamento considerado para o mercado da reserva de potência é o denominado de "reserva atribuída"(Allen and Ilic, 2000), sendo realizados testes para a avaliação do impacto no custo de potência reativa. Vários cenários são simulados e discutidos incluindo casos de contingências.

\section{SUPORTE DE POTÊNCIA REATIVA}

O gerador é um fornecedor de potência ativa e reativa limitada por sua curva de capacidade, como mostrado na Figura 1. A partir desta figura, definem-se as seguintes variáveis:

RP: reserva de potência; $\triangle \mathrm{P}$ : decréscimo da potência programada requerida para aumentar a potência reativa; (Q1,P1), (Q2,P2), (Q3,P3), (Q4,P4) e (Q5,P5): são os pontos de quebra da linearização da curva de capacidade do gerador.

São também definidas as seguintes regiões:

Região $0-Q_{A}$ : região de fornecimento de potência reativa obrigatória $^{1}$ (FPR obrigatória);

\footnotetext{
${ }^{1}$ Região em que a potência reativa é fornecida de zero até certo valor
} 
Região $0-Q_{A}^{\prime}$ : região de absorção de potência reativa obrigatória $^{2}$ (APR obrigatória);

Região $Q_{A}-Q_{B}$ : região de fornecimento extra de potência reativa $^{3}$ (FPR Extra);

Região $Q_{A}^{\prime}-Q_{B}^{\prime}$ : região de absorção extra de potência reativa $^{4}$ (APR Extra);

Região $Q_{B}-Q_{C}$ : região de fornecimento de potência reativa comprometendo a reserva ${ }^{5}$ (FPR Reserva);

Região $Q_{B}^{\prime}-Q_{C}^{\prime}$ : região de absorção de potência reativa comprometendo a reserva ${ }^{6}$ (APR Reserva);

Região $Q_{C}-Q_{D}$ : região de fornecimento de potência reativa com custo de oportunidade ${ }^{7}$ (FPR Oportunidade);

Região $Q_{C}^{\prime}-Q_{D}^{\prime}$ : região de absorção de potência reativa com custo de oportunidade ${ }^{8}$ (APR Oportunidade).

Para uma potência programada $P_{I}$, o suporte de potência reativa é limitado pela curva de capacidade, como ilustrado na Figura 1. Observe-se que o gerador pode injetar potência reativa, mantendo sua potência programada $P_{I}$ no intervalo $\left(0, P_{I}\right)$ até $\left(Q_{B}, P_{I}\right)$, onde $Q_{B}$ é o limite de fornecimento extra de potência reativa. Se houver necessidade de mais potência reativa, o gerador incorre em custo de oportunidade no mercado de Reserva de Potência (RP). Nesta região, a reserva de potência diminui, enquanto a potência reativa aumenta até atingir o limite da curva de capacidade $\left(Q_{C}, P_{I}\right)$. Se houver necessidade de ainda mais potência reativa, o gerador deverá diminuir sua produção de potência ativa em um valor $\triangle \mathbf{P}$ para aumentar a produção de potência reativa até $\left(Q_{D}, P_{F}\right)$, incorrendo num custo de oportunidade no mercado de energia. O gerador deve ser compensado com um pagamento pela perda de oportunidade por usar seu recurso principal para outro propósito.

\subsection{Custo da Reserva de Potência}

A reserva de potência é um produto fundamentalmente diferente da energia. Enquanto que para a energia negociada sua utilização é programada antecipadamente, a reserva de

proporcional à potência ativa programada.

${ }^{2}$ Região em que a potência reativa é absorvida de zero ate certo valor proporcional à potência ativa programada.

${ }^{3}$ Região em que a potência reativa é fornecida além do montante obrigatório, onde o limite é um valor que não comprometa a reserva de potência.

${ }^{4}$ Região em que a potência reativa é absorvida além do montante obrigatório, onde o limite é um valor que não comprometa a reserva de potência.

${ }^{5}$ Região em que a potência reativa é fornecida comprometendo a reserva de potência.

${ }^{6}$ Região em que a potência reativa é absorvida comprometendo a reserva de potência.

${ }^{7}$ Região em que a potência reativa é fornecida comprometendo a potência programada.

${ }^{8}$ Região em que a potência reativa é absorvida, comprometendo a potência programada.

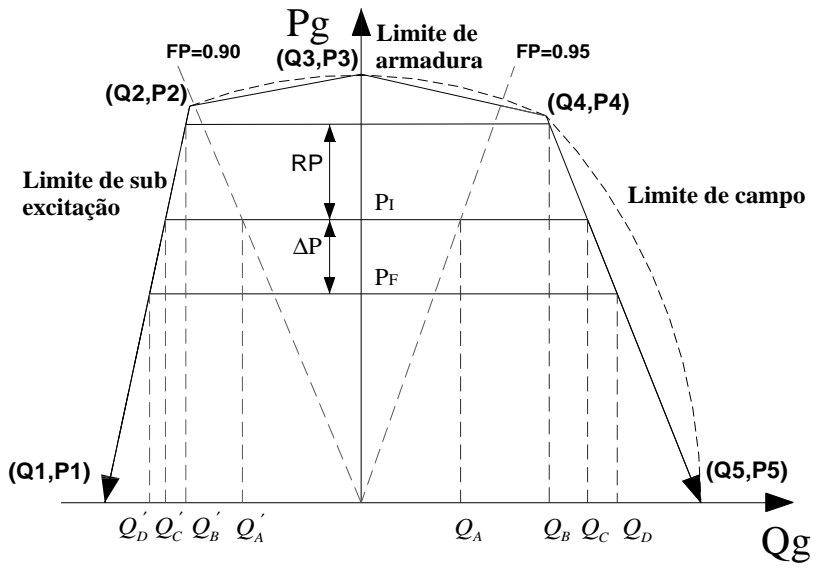

Figura 1: Curva de capacidade do gerador

potência deve estar disponível para ser usada imediatamente, caso ocorra uma contingência no sistema. A oferta para a reserva de potência é feita por geradores, que também atendem o mercado de energia (Allen and Ilic, 2000). A quantidade da reserva de potência negociada depende do nível de confiabilidade e de segurança que se quer. O operador deve calcular e comprar toda a reserva necessária para manter um padrão mínimo de segurança para o sistema. Devido à interdependência existente entre a potência ativa $\mathrm{e}$ reativa, o suporte de potência reativa compromete a reserva de potência em duas regiões do gerador. Nestas regiões, $\left(Q_{B}-Q_{C}\right.$ e $\left.Q_{B}^{\prime}-Q_{C}^{\prime}\right)$ que correspondem ao fornecimento e absorção de potência reativa respectivamente (ver Figura 1), na medida em que o gerador fornece ou consome maior potência reativa, a reserva de potência diminui. O preço da reserva de potência pode ser maior ou menor que o preço da energia, dependendo se os pagamentos por reserva são feitos para a potência ativa utilizada ou para a potência que é simplesmente reservada. Duas formas de compensação econômica para geradores são apresentadas a seguir.

\section{Método 1 - Pagamento pela Potência Suprida}

Neste método, a reserva de potência é remunerada somente se esta é usada realmente. Conseqüentemente, o preço da reserva será maior do que o preço spot (preço do mercado de energia). $O$ gerador recebe um benefício na venda de reserva de potência somente para os períodos quando a reserva é usada e recebe retorno nulo quando não é usada. A formulação deste método é a seguinte.

$\mathrm{CR}=\mathrm{TR} \times \mathrm{PV} \times \mathrm{H}$

onde:

CR : Custo da reserva ( $\$$ )

TR : Tarifa de reserva de potência ( \$/MWh ) 
PV : Potência vendida ( MW )

$\mathrm{H}:$ Tempo de uso (h)

\section{Método 2 - Pagamento por Reserva Atribuída}

Neste método, os agentes de geração recebem pagamento por unidade de reserva em todo o período que esta estiver disponível e recebem compensação diferenciada quando essa reserva estiver sendo utilizada (Allen and Ilic, 2000). A formulação é a seguinte:

$\mathrm{CR}=\mathrm{TR} \times \mathrm{RP} \times \mathrm{Hd}$, se a reserva não for usada (custo por potência)

$\mathrm{CR}=\mathrm{TP} \times \mathrm{PV} \times \mathrm{H}$, se a reserva for usada (custo pela energia)

onde:

CR : Custo da reserva ( $\$$ )

TR : Tarifa de reserva de potência ( $\$ / M W h$ )

TP : Tarifa de mercado ( $\$ / M W h$ )

RP : Reserva de potência ( MW )

$\mathrm{PV}$ : Potência vendida ( MW )

$\mathrm{H}$ : Tempo de uso $(\mathrm{h})$

Hd : Tempo de disponibilidade ( $\mathrm{h}$ )

\subsection{Custo de Perda da Oportunidade}

O OIS (Operador Independente do Sistema) pode requisitar, para garantir uma operação eficiente e segura do sistema, o redespacho de um gerador reduzindo sua potência ativa a fim de permitir que o mesmo forneça ou consuma mais potência reativa; o gerador pode receber um pagamento pelo custo de oportunidade perdido (Cust-Op). O método para calcular este custo é baseado em:

PM : Preço marginal ( \$ )

PI, PF : Potência Inicial e final ( MW )

$\mathrm{f}(\mathrm{p})$ : Curva de custos do gerador

Na Figura 2 é ilustrado o cálculo do custo de oportunidade perdida por um gerador que reduz seu suprimento de potência ativa para fornecer maior suporte de potência reativa. Devido à redução da potência ativa, o gerador receberá menor retorno financeiro. Porém, ao reduzir sua geração, o gerador também diminui o custo de produção da mesma. A redução da renda para o gerador $(\Delta \mathrm{R})$ pode ser descrita através de:

$$
\Delta R=P M \times(P I-P F)-\int_{P I}^{P F} f(p) . d p
$$

O primeiro termo de (1) denota a renda perdida pelo gerador quando sua potência ativa é reduzida de PI até PF. O segundo termo corresponde à redução no custo de produção

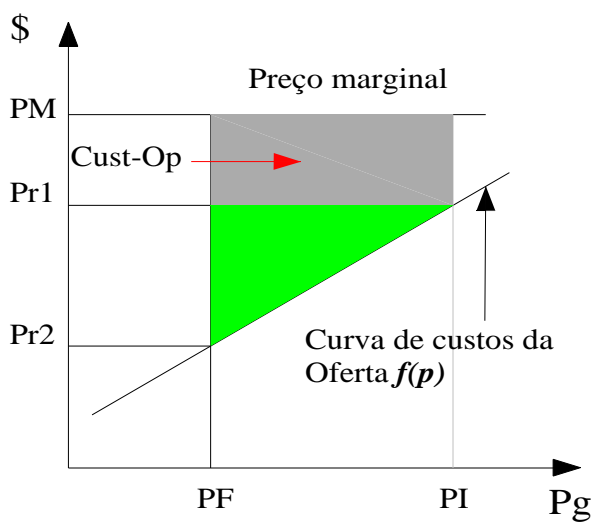

Figura 2: Custo por perda de oportunidade

da geração. A economia do gerador $(\Delta S)$ por reduzir sua potência é descrita por (2), o que corresponde à área do triângulo com vértices en (PF;Pr1), (PF;Pr2) e (PI,Pr1) na Figura 2:

$$
\Delta S=\operatorname{Pr} 1 \times(P I-P F)-\int_{P I}^{P F} f(p) . d p
$$

Portanto, o custo de oportunidade do gerador é a diferença entre (1) e (2):

$$
\text { Cust-Op }=\Delta R-\Delta S
$$

\section{METODOLOGIA PROPOSTA}

\subsection{Estrutura de Custos do Gerador}

Nesta seção é definida a estrutura de custos para cada uma das oito regiões do suporte de potência reativa do gerador síncrono, que foram descritas na seção anterior.

Duas regiões correspondem à obrigatoriedade de fornecer e absorver potência reativa. Em outras duas regiões, o gerador pode ofertar suporte extra de potência reativa sem implicar em prejuízos em outros serviços. Há duas regiões onde o fornecimento de potência reativa limita a oferta de reservas de potência. Finalmente, há duas regiões onde o gerador deve reduzir sua potência ativa programada para uma operação eficiente e segura do sistema através de um maior fornecimento / absorção de potência reativa. Dependendo do ponto de operação do gerador, os agentes geradores farão jus à Compensação Financeira $(\mathrm{CF})$ devido ao atendimento a uma requisição do OIS. Logo, é de grande importância que o OIS tenha acesso aos custos incorridos na prestação deste serviço, de forma que esses cenários possam ser analisados e tomadas as decisões mais econômicas. 
Compensação financeira nas regiões de suporte de potência reativa obrigatório: Estas regiões correspondem aos intervalos $\left(0-Q_{A}\right)$ e $\left(0-Q_{A}^{\prime}\right)$, onde o suporte de potência reativa é obrigatório. $\mathrm{O}$ agente gerador não recebe compensação financeira alguma pelo serviço. Esta região corresponde a pontos de operação onde o fator de potência é maior ou igual a 0,95 e 0,90 para fornecimento e absorção respectivamente, de acordo o padrão da NERC (NERC, online).

Compensação financeira nas regiões de suporte extra de potência reativa: Estas regiões correspondem aos intervalos de $\left(Q_{A}-Q_{B}\right)$ e $\left(Q_{A}^{\prime}-Q_{B}^{\prime}\right)$, onde o agente gerador pode ofertar suporte extra de potência reativa, sem prejudicar a oferta de reserva de potência nem reprogramar a potência ativa. Porém, à medida que aumenta a produção ou absorção de potência reativa, aumentam as perdas de potência ativa no enrolamento do gerador. Logo, o gerador deveria ser compensado pelo custo das perdas ativas adicionais (provavelmente nas horas de pico) e também pela disponibilidade deste serviço.

A determinação de pagamento é muito difícil de ser formulada devido à complexidade envolvida na consignação do pagamento da disponibilidade. Provavelmente, pode ser determinada pelo OIS a partir do impacto no sistema pela não disponibilidade do serviço, isto é, contabilizando o prejuízo de não ter este serviço do gerador. Além disso, o custo das perdas pode ser difícil de ser avaliado devido às variações dos parâmetros das máquinas.

Compensação financeira nas regiões de suporte de potência reativa comprometendo a reserva de potência: Estas regiões correspondem aos intervalos de $\left(Q_{B}-\right.$ $\left.Q_{C}\right)$ e $\left(Q_{B}^{\prime}-Q_{C}^{\prime}\right)$, onde a produção ou absorção de potência reativa só pode ser aumentada se a oferta de reserva de potência ativa é sacrificada. Nestas regiões têm-se maiores perdas ativas no enrolamento do gerador. $\mathrm{O}$ agente gerador deve ser compensado pela disponibilidade de suporte de potência reativa, perdas ativas nos enrolamentos e pela perda da capacidade de ofertar reserva de potência ao sistema.

Compensação financeira nas regiões de suporte de potência reativa comprometendo a produção de energia: Estas regiões correspondem aos intervalos de $\left(Q_{C}-\right.$ $\left.Q_{D}\right)$ e $\left(Q_{C}^{\prime}-Q_{D}^{\prime}\right)$, onde a potência ativa programada precisa ser reduzida a fim de permitir que a unidade produza ou absorva mais potência reativa para atender requisitos de segurança. Além dos custos usuais, o agente gerador deve ser compensado através da tarifa do serviço de fornecimento de potência reativa, a qual deverá considerar o lucro perdido pela diminuição da potência programada, dado por (3).

De acordo com a estrutura de compensação do gerador descrita aqui, é possível generalizar a Compensação
Financeira $(\mathrm{CF})$, o que permite formular matematicamente a estrutura de ofertas, como segue.

Fornecimento de Potência Reativa:

$$
\begin{gathered}
C F\left(Q^{i}\right)=\int_{Q_{A}}^{Q_{B}}[P E R] \cdot d Q+ \\
\int_{Q_{B}}^{Q_{C}}[-C R \times d P / d Q+P E R] \cdot d Q+ \\
\int_{Q_{C}}^{Q_{D}}[-P M \times d P / d Q+P E R] \cdot d Q
\end{gathered}
$$

Absorção de Potência Reativa:

$$
\begin{gathered}
C F\left(Q^{i}\right)=\int_{Q_{B}^{\prime}}^{Q_{A}^{\prime}}[P E R] \cdot d Q+ \\
\int_{Q_{C}^{\prime}}^{Q_{B}^{\prime}}[C R \times d P / d Q+P E R] \cdot d Q+ \\
\int_{Q_{D}^{\prime}}^{Q_{C}^{\prime}}[P M \times d P / d Q+P E R] \cdot d Q
\end{gathered}
$$

Os coeficientes destas equações representam os componentes do custo da potência reativa, e são de responsabilidade do agente gerador provedor deste serviço. O significado desses coeficientes é:

$C F\left(Q^{i}\right)$ : compensação financeira do gerador $\mathrm{i}$;

$P E R$ : preço do suporte extra de potência reativa do gerador;

$d P / d Q$ : derivada de $\mathrm{P}$ com respeito de $\mathrm{Q}$ da curva de capacidade do gerador;

$C R$ : custo da reserva de potência;

$P M$ : preço marginal.

Com a curva de custos dos geradores participantes no serviço ancilar, procede-se a formular o problema como um problema de otimização:

$$
\begin{aligned}
\text { Min } & C F T(Q g)+\Delta P_{\text {Ref }} \times P M \\
= & \sum_{i=1}^{N G} C F\left(Q_{g}^{i}\right)+\Delta P_{\text {Ref }} \times P M
\end{aligned}
$$


sujeito a:

$$
\begin{gathered}
h\left(V, Q_{g}, P_{\text {Prog }}, \Theta\right)=0 \\
V_{\text {Min }} \leq V \leq V^{\text {Max }} \\
Q_{\text {Min }} \leq Q \leq Q^{\text {Max }}
\end{gathered}
$$

onde:

$C F T\left(Q_{g}\right): \quad$ compensação financeira total; $C F\left(Q_{g}^{i}\right)$ : compensação financeira do gerador $i ; \Delta P_{R e f}$ : ajuste da potência na barra de referência para manter o balanço de fluxos; $P M$ : preço marginal $(\$ / \mathrm{Mw}) ; h\left(V, Q_{g}, P_{\text {Prog }}, \theta\right)$ : equações de fluxo de potência; $V_{M i n}, V^{M a x}$ : limites de tensão; $Q_{M i n}, Q^{M a x}$ : limites de potência reativa; $N G$ : número de geradores do sistema.

\subsection{Resolução do problema por estratégias evolutivas}

Dada a não-linearidade das funções envolvidas no problema formulado, as técnicas de inteligência artificial (IA) se tornam atrativas. Neste trabalho, o processo de otimização é resolvido utilizando uma técnica metaheurística, denominada Estratégias Evolutivas (EE). O problema é resolvido pela EE multi-indivíduo ou $(\mu+\lambda)$ - EE. A notação $(\mu+\lambda)$ indica que os $\mu$ novos indivíduos que formarão a nova população de pais são selecionados da união da população de pais com a de descendentes. Note que este mecanismo permite que os melhores indivíduos da população total formem a nova geração. O método é por definição, elitista, pois sempre são favorecidas as melhores soluções (Saavedra, 2005).

São assumidas como variáveis de controle as potências reativas dos geradores. Logo, cada potência reativa gerada pode ser vista como um "gene"(análogo aos algoritmos genéticos) e o vetor de potências reativas geradas como um "cromossomo". Assim, um determinado indivíduo $i$ terá $N G$ genes:

$$
Q i=\left(\left[Q_{i 1}, \ldots, Q_{i j}, \ldots, Q_{i N G}\right],\left[\sigma_{i 1}, \ldots, \sigma_{i j}, \ldots, \sigma_{i N G}\right]\right)
$$

sendo

NG - o número de geradores do sistema;

$Q_{i j}$ - j-ésimo gene do indivíduo i;

$\sigma_{i j}$ - desvio padrão associado ao gene $Q_{i j}$.

Assim, uma população inicial $(Q ; \sigma)$ é formada por vetores indivíduos formando uma estrutura matricial de ordem $\mu \mathrm{x}$ NG, em que a população é inicializada de maneira aleatória, onde $Q_{i j}$ é um valor aleatório, que deve satisfazer a (10) e as restrições de fluxo de potência:

$$
Q_{j}^{\min } \leq Q_{i j} \leq Q_{j}^{\max }
$$

Os desvios padrão são todos inicializados com valores aleatórios no intervalo $[0.0 ; 1.0]$. Na implementação deste trabalho, foi utilizada a Recombinação Discreta (Saavedra, 2005), na qual cada componente do vetor a ser gerado é copiado do componente correspondente de um indivíduo selecionado aleatoriamente da população de pais. Assim, o novo descendente $Q_{k j}$ é produzido:

$$
\mathbf{Q}_{\mathbf{k}, \mathbf{j}}=\operatorname{rand}\left(\begin{array}{c}
Q_{1 j} \\
\vdots \\
Q_{\mu, j}
\end{array}\right), \forall(j=1, \ldots, \mu, e k=1, \ldots, \lambda)
$$

Onde $\operatorname{rand}(\cdot)$ retorna um dos elementos selecionado aleatoriamente, com $\lambda$ sendo o tamanho da população de descendentes. Procedimento idêntico ao mostrado em (11) é feito para a população de desvios padrão. As restrições de fluxo de potência também são satisfeitas neste estágio.

Após o cruzamento, os $\lambda$ descendentes obtidos no passo anterior sofrem um processo de mutação através de uma perturbação Gaussiana. O desvio padrão $(\sigma)$ determina a taxa de mutação da variável objeto (Q). Desse modo, primeiramente deve ser realizado o auto-ajuste dos desvios padrão para em seguida utilizá-los na mutação das variáveis $Q_{i j}$. Esse processo é realizado de acordo com as seguintes expressões:

$$
\begin{gathered}
\sigma_{k j}=\sigma_{k j} \cdot \exp \left(\tau^{\prime} N_{k}(0,1)+\tau N_{j}(0,1)\right) \\
Q_{k j}=Q_{k j}+\sigma_{k j} \times N(0,1) \\
\forall(j=1, \ldots, N, \text { e } k=1, \ldots, \lambda)
\end{gathered}
$$

sendo $\mathrm{N}(0,1)$ um número aleatório com distribuição Gaussiana de média zero e desvio padrão unitário. O fator $\tau^{\prime} N_{k}(0,1)$ permite mudanças globais na mutabilidade, ou seja, todos os genes de determinado indivíduo possuem a mesma taxa de mutação. Já o fator $\tau N_{j}(0,1)$ permite mudanças individuais, isto é, cada gene tem sua própria taxa de mutação. Os fatores $\tau$ e $\tau^{\prime}$ são definidos com "taxas de aprendizagem" e possuem os valores $\tau=(\sqrt{2 N})^{-1} \mathrm{e}$ $\tau^{\prime}=(\sqrt[4]{4 N})^{-1}$, respectivamente. Estas expressões foram sugeridas por (Back and Hammel, 1997). Neste estágio continua-se controlando a restrição do fluxo de potência. 
Portanto, caso não seja respeitada esta restrição, é gerado um novo indivíduo para substituí-lo.

A avaliação das soluções candidatas determinará quais indivíduos são melhores adaptados para sobreviverem nas gerações futuras. Basicamente, este processo implica na avaliação da função objetivo (fitness) para cada solução candidata. As melhores $\mu$ soluções serão selecionados para a próxima geração. Assim, a função fitness é dada por.

$$
\text { Minf }=f_{\text {perdas }}+f_{\text {custo }}+f_{\text {pena }}
$$

onde:

$$
\begin{gathered}
f_{\text {perdas }}=\Delta P_{\text {Ref }} \times P M \\
f_{\text {custo }}=\sum_{i=1}^{N G} C F\left(Q_{g}^{i}\right) \\
f_{\text {Pena }}=\sum_{i \in N_{P Q}} \rho_{v i} \cdot\left(V_{i}-V_{i}^{l}\right)^{2}
\end{gathered}
$$

$f_{\text {perdas }}$ : custo de perdas elétricas no sistema; $N G$ : número de geradores; $\Delta P_{R e f}$ : ajuste da potência na barra de referência para manter o balanço de fluxos; $P M$ : preço marginal; $(\$ / \mathrm{Mw}) ; N_{P Q}$ : número de barras tipo PQ do sistema; $C F\left(Q_{g}^{i}\right)$ : compensação financeira esperada do gerador $i$ pelo suporte de potência reativa; $\rho_{v i}$ : fator de penalização por violação de tensão e $V_{i}^{l}$ : limite violado. $\mathrm{O}$ critério de parada utilizado para este algoritmo é o número máximo de gerações (iterações).

\section{ESTUDO DE CASOS}

Para ilustrar a metodologia e facilitar a análise dos resultados, neste trabalho foram utilizados os sistemas-teste IEEE 14-barras e IEEE 30-barras, em que as impedâncias e demandas foram alteradas (aumentadas).

Para determinar o impacto da reserva de potência no suporte de potência reativa suprido pelos geradores, são realizadas simulações com seis cenários (S1-S6) onde é alterado o preço da reserva de potência, mantendo-se constante o preço da potência reativa extra em 3 \$/Mvar, assim como o preço do mercado de energia em 20 \$/MW. No cenário $\mathrm{S} 1$, o preço da reserva de potência é nulo, enquanto que para os cenários $\mathrm{S} 2, \mathrm{~S} 3, \mathrm{~S} 4, \mathrm{~S} 5$ e S6, o preço da reserva de potência é 3, $5,7,9$, e 12 \$/MW, respectivamente. Para o sistema IEEE 30-barras, as tensões mínimas e máximas assumidas são 0,97 e 1,03 pu, e para o sistema IEEE 14-barras as tensões mínimas e máximas são 0,97 e 1,05 pu, respectivamente. $\mathrm{O}$ desequilíbrio da potência é alocado na barra de folga.

Estudos foram realizados com os dois sistemas- teste, tanto para o caso-base como para uma contingência.

\subsection{Testes com o Sistema IEEE 14-Barras}

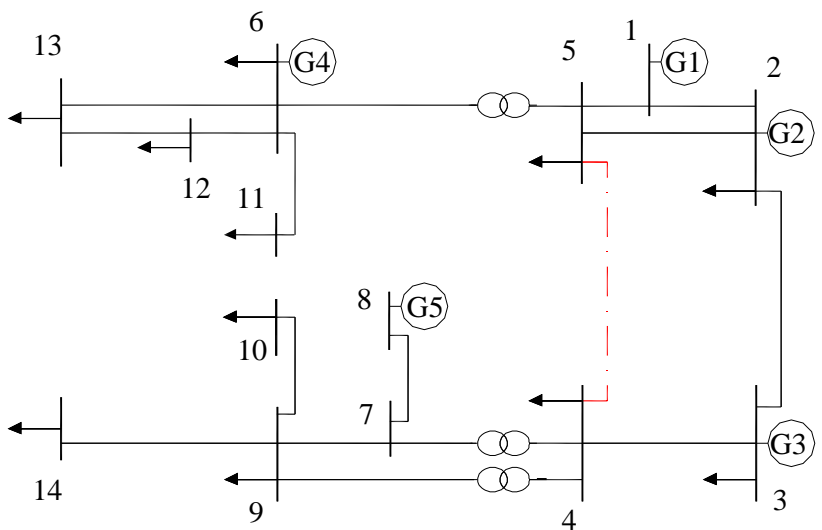

Figura 3: Diagrama Unifilar do Sistema IEEE 14-Barras

\subsubsection{Caso-Base}

O sistema IEEE 14-barras, cujo diagrama unifilar é mostrado na Figura 3, possui 5 geradores, 14 barras, 17 ramos, com uma demanda total de potência ativa e reativa de $\mathbf{2 9 5 , 0 0}$ MW e 140,40 Mvar respectivamente. Neste sistema, a contingência simulada é a saída da linha entre as barras 4 e 5 .

Para a análise deste sistema, adotou-se a linearização por partes da curva de capacidade dos geradores do sistema, onde 5 pares de pontos $(\mathrm{P}, \mathrm{Q})$ representam a curva, como mostrado na Figura 1. Os pontos são apresentados na Tabela 1 para cada um dos geradores do sistema (para simplificar e um melhor entendimento da metodologia, assume-se que todos os geradores têm a mesma curva de capacidade).

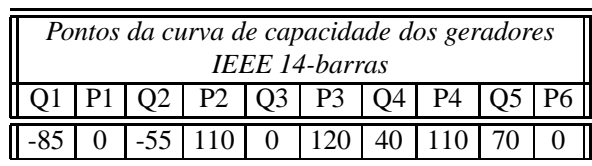

Tabela 1: Pontos da curva de capacidade dos geradores, IEEE 14-barras

Assumem-se os mercados de energia e reserva fechados, isto é, as necessidades contratadas, portanto o despacho dos geradores é conhecido e apresentado na Tabela 2, assim como o preço do mercado, o preço da reserva de potência e o preço do suporte extra de potência reativa.

Com a curva de capacidade dos geradores, as potências programadas e o nível de reservas conhecido, podem ser determinadas as regiões de potência reativa dos geradores. São apresentadas em duas tabelas, onde a Tabela 3 


\begin{tabular}{|c|c|c|c|}
\hline \hline Gerador & Barra & Potência Programada & Reserva de Potência \\
\hline \hline G1 & 1 & $27,25 \mathrm{MW}$ & $70 \mathrm{MW}$ \\
\hline G2 & 2 & $23,40 \mathrm{MW}$ & $70 \mathrm{MW}$ \\
\hline G3 & 3 & $94,60 \mathrm{MW}$ & $10 \mathrm{MW}$ \\
\hline G4 & 6 & $70,00 \mathrm{MW}$ & $40 \mathrm{MW}$ \\
\hline G5 & 8 & $83,40 \mathrm{MW}$ & $25 \mathrm{MW}$ \\
\hline \hline
\end{tabular}

Tabela 2: Programação da potência ativa e reserva de potência, IEEE 14-barras

corresponde ao fornecimento da potência reativa e a Tabela 4 corresponde à absorção de potência reativa.

\begin{tabular}{|c|c|c|c|c|c|c|}
\hline \multirow{2}{*}{\begin{tabular}{|c} 
Gerador \\
$N^{0}$ \\
\end{tabular}} & \multicolumn{2}{|c|}{$\begin{array}{c}\text { Programação } \\
(M W)\end{array}$} & \multicolumn{4}{|c|}{$\begin{array}{c}\text { Limites das regiões de fornecimento } \\
\text { potência reativa (Mvar) }\end{array}$} \\
\hline & $\begin{array}{c}\text { Potência } \\
(M W)\end{array}$ & $\begin{array}{c}\text { Reserva } \\
(M W)\end{array}$ & $\begin{array}{l}\text { FPR } \\
\text { extra }\end{array}$ & $\begin{array}{c}\text { FPR } \\
\text { Reserva }\end{array}$ & $\begin{array}{r}F P 1 \\
\text { oportun }\end{array}$ & \\
\hline G1 & 27,25 & 70 & \begin{tabular}{|l|l|}
8,786 & 43,619 \\
\end{tabular} & \begin{tabular}{|l|l|}
43,619 & 62,710 \\
\end{tabular} & 62,710 & 70 \\
\hline$\overline{\mathrm{G} 2}$ & 23,40 & 70 & \begin{tabular}{l|l}
7,691 & 44,527
\end{tabular} & \begin{tabular}{|l|l|}
$44,52,618$ \\
\end{tabular} & 63,618 & 70 \\
\hline G3 & 94,60 & 10 & \begin{tabular}{l|l|l}
31,09 & 41,473 \\
\end{tabular} & \begin{tabular}{|l|l|}
41,473 & 44,200 \\
\end{tabular} & 44,200 & $\overline{70}$ \\
\hline $\mathrm{G} 4$ & 70,00 & 40 & \begin{tabular}{l|l|}
23,01 & 40,000 \\
\end{tabular} & \begin{tabular}{|l|l|}
40,000 & 50,909 \\
\end{tabular} & 50,909 & 70 \\
\hline G5 & 83,40 & 25 & \begin{tabular}{|l|l|}
27,41 & 40,436 \\
\end{tabular} & \begin{tabular}{|l|l|}
40,436 & 47,255 \\
\end{tabular} & 47,255 & 70 \\
\hline
\end{tabular}

Tabela 3: Limites das regiões de fornecimento de potência reativa - IEEE 14-barras

\begin{tabular}{|c|c|c|c|c|c|c|c|c|}
\hline \multirow{3}{*}{\begin{tabular}{|c|} 
Gerador \\
$N^{0}$ \\
\end{tabular}} & \multicolumn{2}{|c|}{$\begin{array}{c}\text { Programação } \\
(M W)\end{array}$} & \multicolumn{6}{|c|}{$\begin{array}{c}\text { Limites das regiões de absorção de } \\
\text { potência reativa (Mvar) }\end{array}$} \\
\hline & \multicolumn{2}{|c|}{\begin{tabular}{|c|c} 
Potência & Reserva \\
$(M W)$ & $(M W)$
\end{tabular}} & \multicolumn{2}{|c|}{$\begin{array}{l}\text { APR } \\
\text { extra }\end{array}$} & \multicolumn{2}{|c|}{$\begin{array}{c}\text { APR } \\
\text { Reserva }\end{array}$} & \multicolumn{2}{|c|}{$\begin{array}{c}A P R \\
\text { oportunidade }\end{array}$} \\
\hline & 27,25 & 70 & $-12,95$ & $-58,62$ & $-58,62$ & $-77,71$ & $|-77,71|$ & -85 \\
\hline G2 & 23,40 & 70 & $-11,33$ & $-59,53$ & $-59,53$ & $-78,62$ & $-78,62$ & -85 \\
\hline G3 & 94,60 & 10 & $-45,82$ & $-56,47$ & $-56,47$ & $-59,20$ & $-59,20$ & -85 \\
\hline G4 & 70,00 & 40 & $-33,90$ & $-55,00$ & $-55,00$ & $-65,91$ & $-65,91$ & -85 \\
\hline G5 & 83,40 & 25 & $-40,39$ & $-55,44$ & $-55,44$ & $-62,25$ & $-62,25$ & -85 \\
\hline
\end{tabular}

Tabela 4: Limites das regiões de absorção de potência reativa - IEEE 14-barras

Procede-se a minimizar o custo do suporte de potência reativa, considerando as regiões de operação do suporte de potência reativa e os limites de tensão. Após a otimização do custo de suporte de potência reativa para todos os cenários do caso-base (S1, S2, S3, S4, S5, S6), são obtidos os custos e as potências reativas para cada gerador.

As simulações para os diferentes cenários no caso-base são apresentadas na Tabela 5. Nestes casos incorre-se em custo de oportunidade no mercado de reserva de potência. A análise dos resultados mostra que os geradores G1, G2, G3 apresentam alterações consideráveis em seus níveis de geração de potência reativa para cada cenário, à medida que o preço da reserva de potência aumenta. Devido à proximidade elétrica com a demanda de potência reativa, estes três geradores intercambiam entre si o atendimento à carga, procurando minimizar o custo total. Por outro lado, os geradores G4 e G5 têm comportamento independentemente,

\begin{tabular}{|c|c|c|c|c|c|c|c|}
\hline \multirow{2}{*}{\multicolumn{2}{|c|}{$\begin{array}{l}\text { Geradores } \\
\text { do sistema }\end{array}$}} & \multicolumn{6}{|c|}{ Cenários do caso-base, IEEE 14-barras } \\
\hline & & S1 & $S 2$ & $S 3$ & $S 4$ & $S 5$ & S6 \\
\hline \multirow[t]{2}{*}{ G1 } & Custo & 2,76 & 0,05 & 0,00 & 0,00 & 0,00 & 0,00 \\
\hline & Potência re & 9,67 & 8,79 & 8,66 & 5,73 & 3,51 & $-2,85$ \\
\hline \multirow[t]{2}{*}{$\mathrm{G} 2$} & Custo & 33,24 & 28,80 & 49,41 & 48,81 & 71,52 & 86,25 \\
\hline & Potência rea & 18,77 & 17,29 & 24,16 & 23,96 & 31,53 & 36,44 \\
\hline \multirow[t]{2}{*}{ G3 } & Custo & 11,96 & 26,54 & 0,23 & 17,87 & 1,01 & 14,18 \\
\hline & Potência reativa & 35,08 & 39,94 & 31,17 & 37,05 & 31,43 & 35,82 \\
\hline \multirow[t]{2}{*}{ G4 } & Custo & 109,27 & $\overline{162,72}$ & 193,80 & 210,08 & $\overline{248,50}$ & 251,14 \\
\hline & Potência reativa & 45,30 & 45,08 & 44,87 & 44,34 & 44,49 & 43,64 \\
\hline \multirow[t]{2}{*}{ G5 } & Custo & 10,31 & 3,89 & 10,70 & 4,46 & 4,73 & 0,00 \\
\hline & Potência reativa & 30,85 & 28,71 & 30,98 & 28,90 & 28,99 & 27,07 \\
\hline \multirow[t]{2}{*}{ Total } & $\overline{\text { Custo }}$ & $\overline{167,54}$ & 222,00 & $\overline{254,14}$ & $\overline{281,22}$ & $\overline{325,76}$ & $\overline{351,57}$ \\
\hline & Potência reativa & 139,67 & 139,81 & 139,84 & 139,98 & 139,9 & 140,12 \\
\hline
\end{tabular}

Tabela 5: Suporte de potência reativa e seus respectivos custos, IEEE 14-barras

isto é, cada um é responsável pelo atendimento uma determinada área de influência elétrica do ponto de vista de tensão. Portanto, a geração de potência reativa destas unidades mantém-se praticamente constante, embora o custo varie de cenário em cenário.

$\mathrm{O}$ custo total do suporte de potência reativa aumenta à medida que o preço da reserva aumenta. Isto acontece porque, para manter o perfil de tensão dentro dos limites estabelecidos, o gerador G4 aumenta a produção de potência reativa reduzindo o nível de reserva de potência, sendo repassado o custo de oportunidade perdida para o suporte de potência reativa.

\subsubsection{Caso-Contingência}

Considerando-se uma contingência (saída da linha entre as barras 4 e 5), isto provocará um desequilíbrio de fluxos, uma vez que o sistema reage de forma natural pelas leis de Kirchhoff. Neste estado, as novas tensões do sistema podem extrapolar seus limites, como conseqüência da atuação dos controles existentes nos geradores para não terem violadas suas curvas de capacidade. Nessa situação, procede-se a um redespacho de potência reativa.

A potência reativa é redespachada minimizando-se o custo total deste serviço, visando manter os limites de tensão e os geradores trabalhando dentro da curva de capacidade. Os resultados para os diferentes cenários, são mostrados na Tabela 6.

Como pode ser observado, os geradores G3 e G5 diminuíram sua produção de potência reativa devido à contingência. $\mathrm{O}$ déficit de potência reativa no sistema é agora suprido pelos geradores G1, G2 e G4, sendo que G4 em maior proporção, pela natureza local da potência reativa.

Na Figura 4 pode-se observar que o custo total de potência 


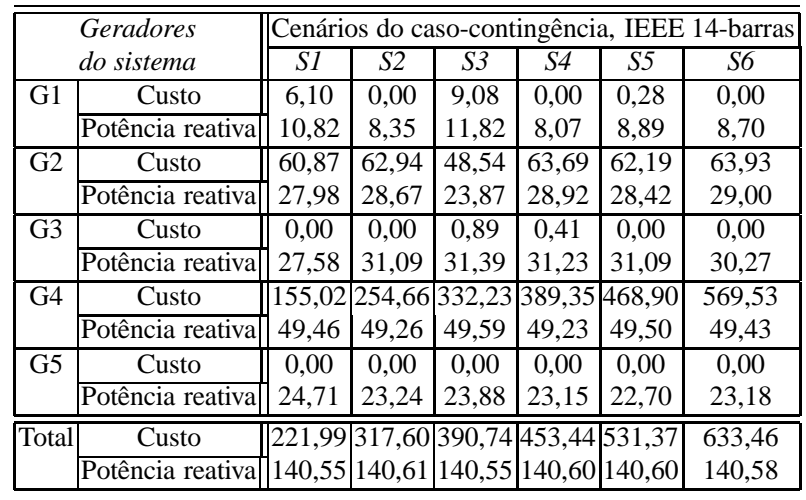

Tabela 6: Suporte reativo e seus respectivos custos, casocontingência, IEEE 14-barras

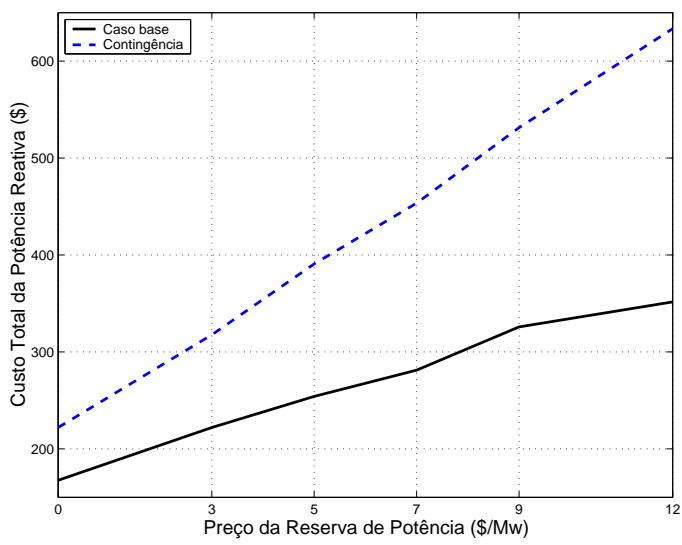

Figura 4: Custo total de potência reativa, IEEE 14-barras, caso-base e caso-contingência

reativa aumenta à medida que o preço da reserva de potência também aumenta, tanto para o caso-base como para o casocontingência. Este aumento no custo da potência reativa é totalmente atribuído ao gerador $\mathrm{G} 4$ porque este deve diminuir seu nível de reserva de potência, para poder suprir potência reativa adicional (para manter o perfil de tensão do sistema), repassando os custos pela perda de oportunidade para o mercado de potência reativa.

\subsection{Testes com o Sistema IEEE 30-Barras}

\subsubsection{Caso-Base}

O sistema IEEE 30-barras, cujo diagrama unifilar é mostrado na Figura 5, possui 6 geradores, 41 ramos e 2 compensadores estáticos, com uma demanda total de potência ativa e reativa de 402,40 MW e 196,20 Mvar respectivamente. A contingência simulada é a saída da linha que se encontra entre as barras 16 e 17 .

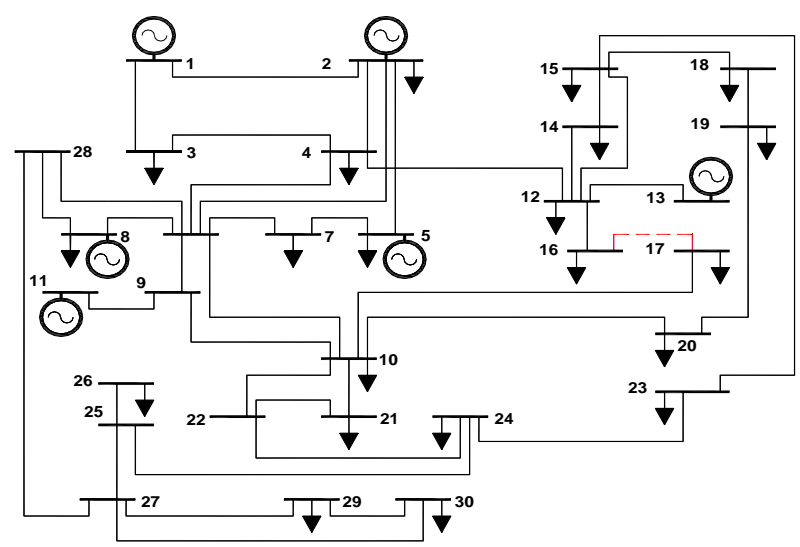

Figura 5: Diagrama Unifilar do Sistema IEEE 30-Barras

Neste sistema também adotou-se a linearização por partes da curva de capacidade dos geradores do sistema. Os 5 pares de pontos que representam as curvas são apresentados na Tabela 7 (assume-se, mais uma vez, que todos os geradores tem a mesma curva de capacidade).

\begin{tabular}{|l|c|c|c|c|c|c|c|c|c||}
\hline \hline \multicolumn{10}{||c||}{ Pontos da curva de capacidade dos geradores } \\
\hline $\mathrm{Q} 1$ & $\mathrm{P} 1$ & $\mathrm{Q} 2$ & $\mathrm{P} 2$ & $\mathrm{Q} 3$ & $\mathrm{P} 3$ & $\mathrm{Q} 4$ & $\mathrm{P} 4$ & $\mathrm{Q} 5$ & $\mathrm{P} 6$ \\
\hline \hline-85 & 0 & -80 & 165 & 0 & 170 & 60 & 110 & 65 & 0 \\
\hline
\end{tabular}

Tabela 7: Pontos da curva de capacidade dos geradores, IEEE 30-barras

De acordo com a metodologia proposta, são conhecidas as potências despachadas e de reserva, assim como o preço das mesmas. A programação dos geradores é apresentada na Tabela 8.

Com estes dados iniciais, encontram-se as regiões de potência reativa na curva de capacidade dos geradores. As regiões de fornecimento são apresentadas na Tabela 9 e as regiões de absorção são apresentadas na Tabela 10.

\begin{tabular}{|c|c|c|c|}
\hline \hline Gerador & Barra & Potência Programada & Reserva de Potência \\
\hline \hline G1 & 1 & $39,73 \mathrm{MW}$ & $80 \mathrm{MW}$ \\
\hline G2 & 2 & $75,20 \mathrm{MW}$ & $80 \mathrm{MW}$ \\
\hline G3 & 5 & $104,3 \mathrm{MW}$ & $50 \mathrm{MW}$ \\
\hline G4 & 8 & $68,10 \mathrm{MW}$ & $90 \mathrm{MW}$ \\
\hline G5 & 11 & $68,50 \mathrm{MW}$ & $90 \mathrm{MW}$ \\
\hline G6 & 13 & $51,90 \mathrm{MW}$ & $100 \mathrm{MW}$ \\
\hline \hline
\end{tabular}

Tabela 8: Programação da potência ativa e reserva de potência, IEEE 30-barras

Procede-se a minimizar o custo do suporte de potência reativa, considerando as regiões de operação e os limites de tensão. Os resultados, nos diferentes cenários, são apresentados na Tabela 11. 


\begin{tabular}{|c|c|c|c|c|c|c|c|c|}
\hline \multirow{2}{*}{\begin{tabular}{|c|} 
Gerador \\
$N^{0}$ \\
\end{tabular}} & \multicolumn{2}{|c|}{$\begin{array}{c}\text { Programação } \\
(M W)\end{array}$} & \multicolumn{6}{|c|}{$\begin{array}{c}\text { Limites das regiões de fornecimento } \\
\text { potência reativa (Mvar) }\end{array}$} \\
\hline & $\begin{array}{c}\text { Potência } \\
(M W)\end{array}$ & $\begin{array}{c}\text { Reserva } \\
(M W)\end{array}$ & \multicolumn{2}{|c|}{$\begin{array}{l}\text { FPR } \\
\text { extra }\end{array}$} & \multicolumn{2}{|c|}{$\begin{array}{c}\text { FPR } \\
\text { Reserva }\end{array}$} & \multicolumn{2}{|c|}{$\begin{array}{c}\text { FPR } \\
\text { oportunidade }\end{array}$} \\
\hline G1 & 39,73 & 80 & 14,39 & 61,25 & 61,25 & \begin{tabular}{|l|}
63,67 \\
\end{tabular} & 63,67 & 65 \\
\hline G2 & 75,20 & 80 & 24,71 & 60,29 & 60,29 & 62,72 & 62,72 & 65 \\
\hline G3 & 104,3 & 50 & 34,28 & 60,32 & 60,32 & 61,83 & 61,83 & 65 \\
\hline G4 & $\begin{array}{l}68,10 \\
\end{array}$ & 90 & 22,38 & 60,20 & 60,20 & 62,93 & 62,93 & 65 \\
\hline G5 & 68,50 & 90 & 22,51 & 60,19 & 60,19 & 62,92 & 62,92 & 65 \\
\hline G6 & 51,90 & 100 & 17,05 & 60,39 & 60,39 & 63,42 & 63,42 & 65 \\
\hline
\end{tabular}

Tabela 9: Limites das regiões de fornecimento de potência reativa, IEEE 30-barras

\begin{tabular}{|c|c|c|c|c|c|c|c|c|}
\hline \multirow{3}{*}{\begin{tabular}{|c|} 
Gerador \\
$N^{0}$ \\
G1
\end{tabular}} & \multicolumn{2}{|c|}{$\begin{array}{c}\text { Programação } \\
(M W)\end{array}$} & \multicolumn{6}{|c|}{$\begin{array}{c}\text { Limites das regiões de absorção de } \\
\text { potência reativa (Mvar) }\end{array}$} \\
\hline & \multicolumn{2}{|c|}{\begin{tabular}{|c|c|} 
Potência & Reserva \\
$(M W)$ & $(M W)$ \\
\end{tabular}} & \multicolumn{2}{|c|}{$\begin{array}{l}\text { APR } \\
\text { extra }\end{array}$} & \multicolumn{2}{|c|}{$\begin{array}{c}\text { APR } \\
\text { Reserva }\end{array}$} & \multicolumn{2}{|c|}{$\begin{array}{c}A P R \\
\text { oportunidade }\end{array}$} \\
\hline & 39,73 & 80 & $-21,20$ & $-81,25$ & $-81,25$ & $-83,67$ & $-83,67 \mid$ & -85 \\
\hline G2 & 75,20 & 80 & $-36,42$ & $-80,30$ & $-80,30$ & $-82,72$ & $-82,72$ & -85 \\
\hline G3 & 104,3 & 50 & $-50,51$ & $-80,32$ & $-80,32$ & $-81,84$ & $-81,84$ & -85 \\
\hline G4 & 68,10 & 90 & $-32,98$ & $-80,21$ & $-80,21$ & $-82,94$ & $-82,94$ & -85 \\
\hline G5 & $\begin{array}{l}68,50 \\
\end{array}$ & 90 & $-33,18$ & $-80,20$ & $-80,20$ & $-82,92$ & $-82,92$ & -85 \\
\hline G6 & 51,90 & 100 & $-25,14$ & $-80,40$ & $-80,40$ & $-83,43$ & $-83,43$ & -85 \\
\hline
\end{tabular}

Tabela 10: Limites das regiões de absorção de potência reativa, IEEE 30-barras

\begin{tabular}{|c|c|c|c|c|c|c|c|}
\hline \multirow{2}{*}{\multicolumn{2}{|c|}{$\begin{array}{l}\text { Geradores } \\
\text { do sistema }\end{array}$}} & \multicolumn{6}{|c|}{ Cenários do caso-base, IEEE 30-barras } \\
\hline & & S1 & $S 2$ & $S 3$ & $S 4$ & S5 & S6 \\
\hline \multirow[t]{2}{*}{ G1 } & Custo & 78,74 & 72,94 & 69,82 & 75,11 & 69,04 & 77,47 \\
\hline & Potênc & 5,47 & $-43,54$ & $-42,48$ & $-44,26$ & $-42,24$ & $-45,05$ \\
\hline \multirow[t]{2}{*}{$\mathrm{G} 2$} & & 00 & 0,00 & 0,00 & 0,00 & 0,00 & 0,00 \\
\hline & Potên & 3,72 & 19,54 & 17,00 & 20,68 & 16,10 & 21,63 \\
\hline \multirow[t]{2}{*}{ G3 } & & & 10 & 31 & 23,87 & 27,74 & 22,73 \\
\hline & Potência re & 40,51 & 41,65 & 44,72 & 42,24 & 43,53 & 41,86 \\
\hline \multirow[t]{2}{*}{ G4 } & $\mathrm{C}$ & & 95,63 & 89,15 & 92,24 & 97,10 & 94,31 \\
\hline & Potênc & & 54,26 & 52,1 & 53,13 & 54,75 & 53,82 \\
\hline \multirow[t]{2}{*}{$\overline{\mathrm{G} 5}$} & $\bar{C}$ & 112,79 & 113,03 & 112,79 & 112,67 & 110,84 & $\overline{111,1}$ \\
\hline & Potênc & 60,11 & 60,19 & & 60,07 & 59,46 & 59,55 \\
\hline \multirow[t]{2}{*}{ G6 } & Custo & & & & 113,46 & 114,12 & 113,37 \\
\hline & Potência reativa & 54,89 & 54,69 & & 54,88 & 55,10 & 54,85 \\
\hline & & \multirow{2}{*}{\multicolumn{2}{|c|}{\begin{tabular}{|l|l|}
415,53 & 416,59 \\
186,75 & 186,79
\end{tabular}}} & & & & \\
\hline & & & & & & & \\
\hline
\end{tabular}

Tabela 11: Suporte de potência reativa e seus respectivos custos, caso-base, IEEE 30-barras

Observando os resultados obtidos para estes cenários, verifica-se que para manter o perfil de tensão dentro dos limites requeridos, foi necessário que todos os geradores (exceto G2, que permaneceu na região de fornecimento de potência reativa obrigatório), passaram a fornecer/absorver potência reativa na região de suporte extra de potência reativa. Entretanto, os perfis de suporte de potência reativa obtidos de um cenário para outro ficaram relativamente próximos assim como o custo total deste serviço.

Em conclusão, em nenhum dos cenários relativos ao caso- base, os geradores incorreram em custos por perda de oportunidade nos mercados de reserva de potência e de energia. Em outras palavras, o custo do suporte de potência reativa é invariável e independente desses outros mercados, por que há suficiente potência reativa, para atender as necessidades de operação dos cenários analisados.

\subsubsection{Caso-Contingência}

A contingência simulada foi a saída da linha entre as barras 16 e 17, a qual causa violações de limites de tensão. Redespacha-se a potência reativa, com o objetivo de minimizar o custo total do suporte de potência reativa, visando manter os limites de tensão e os limites da curva de capacidade. Os resultados para os cenários simulados são apresentados na Tabela 12.

\begin{tabular}{|c|c||c|c|c|c|c|c|}
\hline \hline \multicolumn{3}{|c||}{$\begin{array}{l}\text { Geradores } \\
\text { do sistema }\end{array}$} & \multicolumn{6}{c|}{ Cenários do caso-contingência, IEEE 30-barras } \\
\cline { 2 - 8 } & $S 1$ & $S 2$ & $S 3$ & $S 4$ & $S 5$ & $S 6$ \\
\hline G1 & Custo & 91,10 & 110,81 & 104,62 & 91,42 & 88,46 & 73,22 \\
& Potência reativa & $-51,57$ & $-59,56$ & $-56,15$ & $-54,24$ & $-52,74$ & $-47,34$ \\
\hline \multirow{2}{*}{ G2 } & Custo & 7,06 & 41,47 & 11,80 & 15,61 & 8,50 & 0,00 \\
& Potência reativa & 27,07 & 38,54 & 28,65 & 29,92 & 27,55 & 13,08 \\
\hline G3 & Custo & 21,41 & 28,37 & 55,88 & 16,25 & 26,60 & 52,79 \\
& Potência reativa & 41,42 & 43,74 & 52,91 & 39,70 & 43,15 & 51,88 \\
\hline G4 & Custo & 102,62 & 86,48 & 77,60 & 110,03 & 98,84 & 101,30 \\
& Potência reativa & 56,59 & 51,21 & 48,25 & 59,06 & 55,33 & 56,15 \\
\hline G5 & Custo & 398,33 & 678,89 & 848,33 & 1048,13 & 1224,17 & 1491,53 \\
& Potência reativa & 63,04 & 63,12 & 63,04 & 63,19 & 63,16 & 63,14 \\
\hline G6 & Custo & 99,90 & 97,68 & 98,70 & 96,51 & 99,93 & 98,61 \\
& Potência reativa & 50,36 & 49,62 & 49,96 & 49,23 & 50,37 & 49,93 \\
\hline \hline \multirow{2}{*}{ Total } & Custo & 720,42 & 1043,71 & 1196,93 & 1377,96 & 1546,51 & 1817,46 \\
& Potência reativa & 186,91 & 186,67 & 186,66 & 186,86 & 186,82 & 186,84 \\
\hline
\end{tabular}

Tabela 12: Suporte reativo e seus respectivos custos, casocontingência: IEEE 30-barras

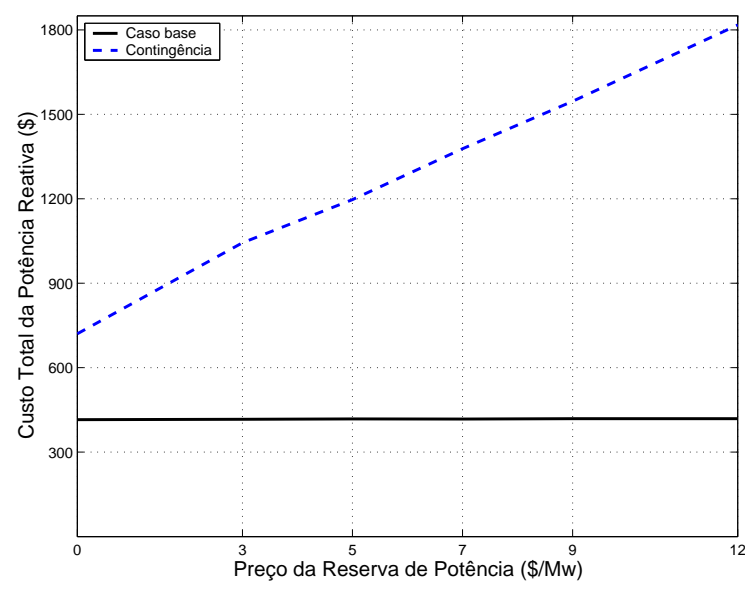

Figura 6: Custo total de potência reativa, IEEE 30-barras, caso-base e caso-contingência

Observa-se que a saída da linha 16-17 prejudica principalmente $\mathrm{o}$ atendimento às barras 10, 20, $21 \mathrm{e}$ 
22. Os fluxos de potência para atendimento à barra 17 deverão seguir outras rotas envolvendo essas barras, reduzindo o perfil de tensão das mesmas. Para recuperar o perfil de tensão e atender os limites estabelecidos, o único gerador com proximidade elétrica é G5. Para sustentar o perfil de tensão dessa área, o gerador deve utilizar toda sua capacidade de potência reativa e ainda incorrer em perda de oportunidade nos mercados de reserva de potência e de energia, como pode ser observado na Tabela 12 .

$\mathrm{Na}$ Figura 6 pode ser observado que, para o casocontingência, o custo do suporte de potência reativa cresce à medida que o preço de reserva de potência aumenta, evidenciando o acoplamento entre esses mercados. Esta situação contrasta com o caso-base, onde o custo do suporte de potência reativa é independentemente desses mercados.

\section{CONCLUSÕES}

Devido à curva de capacidade da máquina síncrona, o provimento do suporte de potência reativa poderá implicar em perda de oportunidade nos mercados de reserva e de energia para um agente gerador.

Por exemplo, quando o gerador trabalha em certo limite da curva de capacidade, um aumento na geração de potência reativa implicaria na necessidade de diminuição da reserva de potência. Deve-se notar ainda que a relação de ordem de grandeza entre estas é grande, por exemplo, para aumentar a potência reativa em 3 Mvar seria necessário reduzir em 10 MW a reserva de potência. Logo, devido a este efeito, o custo da potência reativa nesta região seria elevado. O mesmo fenômeno acontece quando o gerador incorre em custo de oportunidade no mercado de energia.

A metodologia proposta procura no sistema recursos de potência reativa a custo mínimo, levando em consideração o acoplamento existente entre o mercado de energia, reserva de potência e suporte de potência reativa.

Os resultados numéricos dos testes indicam que a necessidade de suporte extra de potência reativa, implica no uso de recursos do mercado de energia e reserva de potência, acarretando em aumento de custo do suporte de potência reativa, consequiência da perda de oportunidade naqueles mercados.

A operação nestas regiões da curva de capacidade, implicando em custos elevados, pode ser interpretada como um indicador de mercado para a instalação de novas fontes de potência reativa no sistema. Antes disso, entretanto, é necessária a definição de políticas de remuneração para este tipo de fonte, de forma a tornar atraente esse tipo de investimento.

\section{REFERÊNCIAS}

Allen, E. and Ilic, M. (2000). Reserve markets for power systems reliability, IEEE Transactions on Power Systems 15: pp. (228-233).

Back, T. and Hammel, U. (1997). Evolutionary computation Comments on the history and current state, IEEE Transactions on Evolutionary Computation 1: pp. (317).

Bhattacharya, K. and Zhong, J. (2001). Reactive power as an ancillary service, IEEE Transactions on Power Systems 18: pp. (294-300).

Gross, G. and Tao, S. (2002). Unbundled reactive support service key characteristics and dominant cost component, IEEE Transactions on Power Systems 17: pp. (283-289).

Hirst, E. and Kirby, B. (1997). Electric power ancillary services, Ridge National Lab. Tech. Rep .

Kundur, P. and Zhang, Y. (2001). Valuation of dynamic reactive power support services for transmission access, IEEE Transactions on Power Systems 16: pp. (719728).

NERC (on-line). Nerc planning standards, North American Electric Reliability Council.//www.nerc.com. Acesso em: 03 jan 2005.

Saavedra, O. (2005). Estratégias evolutivas e programação evolutiva, http://www.dee.ufma.br/ osvaldo/ia.htm. Acesso em: 03 jan 2005 .

Zhong, J. (2003). On some aspects of design of electric power ancillary service markets, Doctoral thesis. Göteborg, Sweden, Chalmers University of Technology, Department of Electric Power Engineering . 